\title{
BMJ Observational cohort study of the safety Open of digoxin use in women with heart failure
}

\author{
James H Flory, ${ }^{1}$ Bonnie Ky, ${ }^{2}$ Kevin Haynes, ${ }^{2}$ Steve M Brunelli, ${ }^{3}$ Jeffrey Munson, ${ }^{4}$ \\ Christopher Rowan, ${ }^{5}$ Brian L Strom, ${ }^{2}$ Sean Hennessy ${ }^{2}$
}

To cite: Flory JH, Ky B, Haynes $\mathrm{K}$, et al.

Observational cohort study of the safety of digoxin use in women with heart failure. BMJ Open 2012;2:e000888. doi:10.1136/

bmjopen-2012-000888

- Prepublication history and additional material for this paper are available online. To view these files please visit the journal online (http://dx. doi.org/10.1136/ bmjopen-2012-000888).

Received 16 January 2012 Accepted 9 March 2012

This final article is available for use under the terms of the Creative Commons Attribution Non-Commercial 2.0 Licence; see http://bmjopen.bmj.com

For numbered affiliations see end of article.

Correspondence to Dr James Howard Flory; jaf9052@nyp.org

\section{ABSTRACT}

Objectives: This study aims to assess whether digoxin has a different effect on mortality risk for women than it does for men in patients with heart failure (HF).

Design: This study uses the UK-based The Health Information Network population database in a cohort study of the impact of digoxin exposure on mortality for men and women who carry the diagnosis of HF. Digoxin exposure was assessed based on prescribing data. Multivariable Cox proportional hazards models were used to assess whether there was an interaction between sex and digoxin affecting mortality hazard.

Setting: The setting was primary care outpatient practices.

Participants: The study cohort consisted of 17707 men and 19227 women with the diagnosis of HF who contributed only time without digoxin exposure and 9487 men and 10808 women with the diagnosis of HF who contributed time with digoxin exposure.

Main outcome measures: The main outcome measure was all-cause mortality.

Results: The primary outcome of this study was the absence of a large interaction between digoxin use and sex affecting mortality. For men, digoxin use was associated with a HR for mortality of 1.00 , while for women, the HR was also 1.00 ( $p$ value for interaction $0.65)$. The results of sensitivity analyses were consistent with those of the primary analysis.

Conclusion: Observational data do not support the concern that there is a substantial increased risk of mortality due to the use of digoxin in women. This finding is consistent with previous observational studies but discordant with results from a post hoc analysis of a randomised controlled trial of digoxin versus placebo.

\section{INTRODUCTION}

It has been hypothesised that digoxin, when used in the treatment of heart failure (HF), may increase mortality by approximately $20 \%$ in women but not in men. This hypothesis is based on a post hoc analysis in 6800 patients by Rathore and colleagues of the Digitalis

\section{ARTICLE SUMMARY}

Article focus

- Digoxin is used in patients with HF and has been shown in one major randomised control trial, the Digitalis Investigation Group study, to reduce the rate of hospitalisations in that population.

- Post hoc analysis of Digitalis Investigation Group indicated that digoxin, when used in the treatment of HF, may increase mortality by approximately $20 \%$ in women but not in men. Further randomised trials evaluating the interaction between digoxin and sex have not emerged.

Key messages

- For men, digoxin use was associated with a HR for mortality of 1.00 , while for women, the HR was also 1.00 ( $p$ value for interaction 0.65 ). There was no evidence of a different association between digoxin use and mortality in women compared with men. Sensitivity analyses did not affect this estimate materially.

- An interesting incidental finding of this study is that interventions known to reduce mortality in $\mathrm{HF}$ are used less in women than in men who have been diagnosed with $\mathrm{HF}$.

Strengths and limitations of this study

- The major strength of this study was its large sample size, which allowed adjustment for many covariates and numerous sensitivity analyses, none of which affected the conclusions.

- The major limitation of this study is that it is nonrandomised. Although there is no evidence of substantial confounding of the main study result, confounding could still bias these results.

Investigation Group trial, a placebocontrolled randomised trial that showed digoxin did not affect overall mortality but did reduce hospitalisations in patients with HF. ${ }^{1}$ The post hoc analysis examined mortality effects by sex and found that compared with placebo digoxin conferred reduced mortality in men (absolute difference $-1.6 \%, 95 \%$ CI $-4.2 \%$ to $1.0 \%)$ and increased mortality in women (absolute difference $4.2 \%, 95 \%$ CI $-0.5 \%$ to $8.8 \%$ ), 
with a statistically significant interaction ( $p$ value $=0.034) .{ }^{2}$ One proposed mechanism is that women may have higher mean serum digoxin levels than men. There is evidence that even within the therapeutic range, higher serum digoxin levels are associated with increased mortality, and experts have argued that maintenance of serum digoxin levels at the low end of the therapeutic range may be the key to safe, effective use of the drug in either sex. ${ }^{3-7}$

Because the sex-digoxin interaction was based on post hoc analyses, it needs cautious interpretation. Yet, if true, the finding is clinically important because digoxin continues to be widely used by both women and men. ${ }^{8}$ It remains unclear whether digoxin should be used differently in the different sexes, and concerns about its use in women continue to appear in the literature. ${ }^{9} 10$ Unwarranted recommendations against use of digoxin in women would deprive a large population of a medicine with demonstrated ability to prevent hospitalisations.

Further randomised trials evaluating the interaction between digoxin and sex have not emerged. One observational study based on the Studies of Left Ventricular Dysfunction cohort found no difference in digoxin's effect on mortality between sexes but concluded that additional research in other populations is still needed. ${ }^{10}$ Limitations of that study included restriction to a population with severely reduced ejection fraction $(<35 \%)$ and relatively small sample size $(n=6797)$. Another observational study performed on 2841 propensity score-matched patients with $\mathrm{HF}$ also did not report any difference in digoxin's effect on mortality between the sexes; this study was primarily limited by a relatively small size and restriction to a single clinical center. ${ }^{11}$ We sought to conduct a much larger study in a broader population that could help assess whether digoxin increases mortality in women compared with men.

\section{METHODS}

\section{Study design}

We conducted a retrospective cohort study to test the primary null hypothesis that sex was not an effect modifier for the association of digoxin use with mortality. We also conducted planned secondary analyses of whether effect modification was mediated by digoxin dose or by serum digoxin concentration. This study was approved by the Institutional Review Board of the University of Pennsylvania. Analyses were conducted using SAS V.9.1.

\section{Study population}

The study used the Health Improvement Network (THIN) Database, a primary care electronic medical record database in the UK. This database contains over 5 million individuals who have contributed person-time from over 300 different general practices from 1986 to 2008. THIN includes demographic information on patients, as well as records of prescribed drugs, medical diagnoses (coded as READ codes), as well as vital signs and laboratory values on a subset of patients. It is a representative subset of the UK's general population. ${ }^{12}$

\section{Cohort definition}

The analysis was restricted to individuals with a diagnostic code for HF. Once that condition was met, patients could contribute follow-up time as long as they were being given regular prescriptions of at least one drug consistent with the treatment for HF (as described below). Information was only used if it was collected during times when the adjusted mortality ratio, a quality control measure used in THIN to identify practices which are recording deaths accurately, was within acceptable standards for a given practice. ${ }^{13}$ It was possible for one individual to contribute both exposed and unexposed time. The result was four cohorts with some within-sex cross-over: men on digoxin, men not on digoxin, women on digoxin and women not on digoxin.

\section{Exposed group}

Exposed subjects were defined as individuals who carried the diagnosis of HF and were receiving two or more consecutive prescriptions for digoxin. Individuals were assumed to be on digoxin from the date of receipt of a prescription until 30 days after their prescription ran out (the intended duration of a prescription was either computed from information on daily dose and number of tablets or imputed as 30 days if that information was not available).

\section{Unexposed group}

Unexposed subjects were defined as individuals who carried a diagnosis of $\mathrm{HF}$ and were receiving two or more consecutive prescriptions for a loop diuretic, $\beta$ blocker, ACE inhibitor or angiotensin receptor blocker. They only contributed person-time to the cohort while receiving these drugs; they were assumed to be on a drug from the date of receipt of a prescription until 30 days after their prescription ran out, which was defined with the same criteria used for digoxin exposure. These are drugs that, while not specific for HF, are commonly used for that condition.

\section{Definition of outcome}

The outcome for this study was all-cause mortality, as documented by a date of death in the THIN demographics file.

\section{Definition of covariates}

Baseline covariates consisted of diagnostic codes for common comorbidities of HF, including hypertension, stroke, myocardial infarction, chronic obstructive pulmonary disease, diabetes and atrial fibrillation. We also assessed age, sex and baseline use of drugs commonly used in HF, notably aspirin, statins, oral anticoagulants, potassium-sparing diuretics and the HFassociated drugs listed above. To be included as 
a baseline variable, drug use had to be in the year prior to HF diagnosis; medical history such as a history of hypertension could be coded at any time prior to $\mathrm{HF}$ diagnosis. Blood pressure, body mass index (BMI) and a variety of laboratory values including serum creatinine were available on a minority of participants and were used as baseline variables in sensitivity analysis. Once a patient began to contribute exposure time, baseline variables were no longer updated.

\section{Statistical analysis}

Because of the large sample size, we anticipated that even clinically insignificant differences between baseline variables could be statistically significant and therefore described baseline variables using standardised differences. ${ }^{14}$ Standardised differences are a balance diagnostic used to assess how similar groups are at baseline-conceptually, they are equal to the difference of a variable's mean between two groups divided by the $\mathrm{SD}$ for that variable. A standardised difference of $<0.1$, by convention, is considered to indicate good balance of groups on that variable. ${ }^{14}$ For categorical variables, a standardised difference between men and women was

calculated as $\frac{p_{\text {women }}-p_{\text {men }}}{\sqrt{\frac{p_{\text {women }}\left(1-p_{\text {women }}\right)+p_{\text {men }}\left(1-p_{\text {men }}\right)}{2}}}$,

where $\mathrm{p}=$ proportion of the population falling into a category. In addition, because the most relevant question was whether these between-sex differences were consistent in both digoxin-exposed and digoxinunexposed groups, this equation was extended to treat the inter-sex differences for the exposed and unexposed groups as variables which in turn had their own standardised differences: inclusion of digoxin dose and then serum digoxin level in the model to assess for any evidence that these variables mediated any interaction. Finally, sensitivity analyses were conducted in which baseline blood pressure, BMI and serum creatinine were included in the analysis, in which the cohort was restricted to participants using concomitant loop diuretics, in which comorbid conditions were defined based only on data from the 1 year preceding cohort entry, in which patients with a diagnosis of atrial fibrillation were excluded, in which data were included even if the adjusted mortality ratio at the time of collection was not acceptable and in which subjects were excluded as soon as they crossed over from one exposure group into another.

\section{RESULTS}

\section{Characteristics of the study population}

The study cohort consisted of 17707 men and 19227 women who contributed time only without digoxin exposure and 9487 men and 10808 women who contributed time with digoxin exposure. Table 1 presents differences in baseline variables between these groups. In general, many variables were differently distributed between sexes, as reflected by high standardised differences. However, sex differences were consistent between digoxin-exposed and unexposed groups, as reflected by low standardised differences of differences $(<0.1$ for all variables).

Baseline drug utilisation data were notable for high rates of loop diuretic use (table 1). Oral anticoagulant use was much more common among digoxin users even after controlling for baseline diagnosis of atrial fibrillation. Examination of trends in drug use during the year after the diagnosis of $\mathrm{HF}$ was documented (figure 1) shows rapid increases in the use of ACE inhibitors,

$\frac{\left[\left(p_{\text {women, exposed }}-p_{\text {men, exposed }}\right)-\left(p_{\text {women, unexposed }}-p_{\text {men, unexposed }}\right)\right]}{\sqrt{\frac{\left[\left(p_{\text {women, exposed }}\left(1-p_{\text {women, exposed }}\right)+p_{\text {men, exposed }}\left(1-p_{\text {men, exposed }}\right)+p_{\text {women, unexposed }}\left(1-p_{\text {women, unexposed }}\right)+p_{\text {men, unexposed }}\left(1-p_{\text {men, unexposed }}\right)\right)\right]}{4}}}$

Total follow-up time for all individuals was assessed, with digoxin use, digoxin dose and serum digoxin concentrations as time-varying covariates in secondary analysis. In the primary analysis, digoxin dose and serum concentration were ignored. A Cox proportional hazards model was used to calculate HRs for sex, digoxin use and the interaction of sex and digoxin use, controlling for the covariates specified above. ${ }^{15}$

The primary parameter of interest was the adjusted HR for the interaction of sex and digoxin use, where a significant deviation from the null would indicate that digoxin use was associated with mortality differently for men than for women. Specifically, a HR for interaction significantly greater than one would indicate that digoxin was associated with greater mortality in women than in men. This analysis was then repeated with $\beta$ blockers and spironolactone, with higher rates of use of all these agents in men compared with women (figure 1A,B).

Blood pressure was recorded for the majority of subjects, while other quantitative covariates such as BMI and serum creatinine were recorded on a minority of subjects. In general, rates of missing data were higher for women than for men. Baseline systolic BP mean $>140$ was considerably more common in women, while a serum creatinine greater than $150 \mathrm{mg} / \mathrm{dl}$ was considerably more common in men.

\section{Digoxin prescribing patterns over time}

We found that digoxin use among subjects in this study remained prevalent at about $25 \%$ throughout the time period covered by the database (figure $1 \mathrm{~A}, \mathrm{~B}$ ). 
Table 1 Demographic and clinical features of cohort

\begin{tabular}{|c|c|c|c|c|c|c|c|}
\hline & \multicolumn{2}{|c|}{$\begin{array}{l}\text { Digoxin } \\
\text { non-use }\end{array}$} & \multirow{2}{*}{$\begin{array}{l}\text { Standardised } \\
\text { difference }\end{array}$} & \multicolumn{2}{|c|}{ Digoxin use } & \multirow{2}{*}{$\begin{array}{l}\text { Standardised } \\
\text { difference }\end{array}$} & \multirow{2}{*}{$\begin{array}{l}\text { Standardised } \\
\text { difference } \\
\text { of differences }\end{array}$} \\
\hline & Male & Female & & Male & Female & & \\
\hline Total (N) & 17707 & 19227 & & 9487 & 10808 & & \\
\hline \multicolumn{8}{|l|}{ Baseline demographics } \\
\hline Age $<50$ & $3 \%$ & $2 \%$ & 0.08 & $2 \%$ & $1 \%$ & 0.11 & 0.01 \\
\hline Age $50-64$ & $16 \%$ & $8 \%$ & 0.25 & $15 \%$ & $5 \%$ & 0.31 & 0.02 \\
\hline Age $65-74$ & $28 \%$ & $22 \%$ & 0.15 & $30 \%$ & $19 \%$ & 0.25 & 0.07 \\
\hline Age $>74$ & $52 \%$ & $68 \%$ & 0.33 & $54 \%$ & $75 \%$ & 0.45 & 0.08 \\
\hline \multicolumn{8}{|l|}{ Baseline medical history } \\
\hline History of MI & $28 \%$ & $15 \%$ & 0.33 & $22 \%$ & $12 \%$ & 0.28 & 0.05 \\
\hline History of stroke & $14 \%$ & $13 \%$ & 0.04 & $14 \%$ & $14 \%$ & 0.00 & 0.03 \\
\hline History of diabetes & $20 \%$ & $15 \%$ & 0.12 & $16 \%$ & $13 \%$ & 0.10 & 0.02 \\
\hline History of hypertension & $44 \%$ & $49 \%$ & 0.10 & $41 \%$ & $45 \%$ & 0.09 & 0.01 \\
\hline History of COPD & $14 \%$ & $11 \%$ & 0.11 & $13 \%$ & $8 \%$ & 0.16 & 0.02 \\
\hline History of fracture & $7 \%$ & $12 \%$ & 0.19 & $6 \%$ & $11 \%$ & 0.18 & 0.01 \\
\hline History of pneumonia & $4 \%$ & $3 \%$ & 0.02 & $4 \%$ & $3 \%$ & 0.05 & 0.02 \\
\hline $\begin{array}{l}\text { History of atrial } \\
\text { fibrillation }\end{array}$ & $10 \%$ & $8 \%$ & 0.09 & $41 \%$ & $41 \%$ & 0.00 & 0.05 \\
\hline \multicolumn{8}{|l|}{ Baseline drug use } \\
\hline Statin user & $25 \%$ & $16 \%$ & 0.23 & $15 \%$ & $10 \%$ & 0.18 & 0.06 \\
\hline Loop diuretic user & $53 \%$ & $56 \%$ & 0.06 & $59 \%$ & $58 \%$ & 0.01 & 0.05 \\
\hline Aspirin user & $42 \%$ & $32 \%$ & 0.21 & $35 \%$ & $30 \%$ & 0.11 & 0.08 \\
\hline Oral anticoagulant user & $9 \%$ & $5 \%$ & 0.15 & $24 \%$ & $18 \%$ & 0.13 & 0.03 \\
\hline Nitrate user & $31 \%$ & $24 \%$ & 0.15 & $26 \%$ & $20 \%$ & 0.13 & 0.02 \\
\hline K-Sparing diuretic user & $20 \%$ & $25 \%$ & 0.13 & $24 \%$ & $29 \%$ & 0.09 & 0.02 \\
\hline Spironolactone user & $5 \%$ & $4 \%$ & 0.04 & $6 \%$ & $5 \%$ & 0.04 & 0.00 \\
\hline$\beta$ Blocker user & $26 \%$ & $22 \%$ & 0.10 & $19 \%$ & $18 \%$ & 0.03 & 0.05 \\
\hline ACE user & $36 \%$ & $28 \%$ & 0.18 & $35 \%$ & $27 \%$ & 0.18 & 0.00 \\
\hline ARB user & $6 \%$ & $6 \%$ & 0.02 & $5 \%$ & $5 \%$ & 0.01 & 0.02 \\
\hline Thiazide user & $16 \%$ & $23 \%$ & 0.18 & $16 \%$ & $22 \%$ & 0.16 & 0.02 \\
\hline CCB user & $27 \%$ & $24 \%$ & 0.08 & $23 \%$ & $21 \%$ & 0.04 & 0.03 \\
\hline \multicolumn{8}{|c|}{ Laboratory data and vital signs } \\
\hline \multicolumn{8}{|c|}{ BMI } \\
\hline$\%$ with data & $36 \%$ & $27 \%$ & 0.20 & $32 \%$ & $24 \%$ & 0.19 & 0.01 \\
\hline $\mathrm{BMI}<18$ & $1 \%$ & $3 \%$ & 0.16 & $1 \%$ & $4 \%$ & 0.21 & 0.05 \\
\hline BMI 18-25 & $27 \%$ & $30 \%$ & 0.07 & $31 \%$ & $37 \%$ & 0.13 & 0.05 \\
\hline BMI 25-30 & $41 \%$ & $31 \%$ & 0.22 & $41 \%$ & $31 \%$ & 0.21 & 0.00 \\
\hline BMI 30-35 & $22 \%$ & $20 \%$ & 0.03 & $19 \%$ & $16 \%$ & 0.06 & 0.02 \\
\hline BMI >35 & $10 \%$ & $16 \%$ & 0.18 & $9 \%$ & $12 \%$ & 0.09 & 0.07 \\
\hline \multicolumn{8}{|l|}{ Creatinine } \\
\hline$\%$ with data & $40 \%$ & $33 \%$ & 0.14 & $33 \%$ & $29 \%$ & 0.09 & 0.04 \\
\hline $\mathrm{Cr}>150$ & $16 \%$ & $8 \%$ & 0.23 & $14 \%$ & $7 \%$ & 0.23 & 0.01 \\
\hline \multicolumn{8}{|l|}{ Blood pressure } \\
\hline$\%$ with data & $71 \%$ & $65 \%$ & 0.12 & $66 \%$ & $61 \%$ & 0.10 & 0.02 \\
\hline $\mathrm{SBP}>140$ & $50 \%$ & $60 \%$ & 0.21 & $47 \%$ & $58 \%$ & 0.22 & 0.01 \\
\hline
\end{tabular}

ARB, angiotensin receptor blocker; BMI, body mass index; CCB, calcium channel blocker; COPD, chronic obstructive pulmonary disease; $\mathrm{Cr}$, creatinine; MI, myocardial infarction; SBP, systolic blood pressure.

Digoxin dose was consistently lower in women than in men (mean daily dose $136 \mu \mathrm{g}$ in women and $159 \mu \mathrm{g}$ in men, $\mathrm{p}<0.001$ ), and the average dose in both groups declined over time by $1.6 \mu \mathrm{g} /$ year in men and $1.3 \mu \mathrm{g}$ / year in women $(\mathrm{p}<0.001$ for both trends but no significant difference in the rate of decline for men vs women).

Serum digoxin levels were available for 8563 separate measurements covering 4063 individuals. The mean serum level for men was $1.2 \mathrm{ng} / \mathrm{dl}$, while for women, it was $1.3 \mathrm{ng} / \mathrm{dl}(\mathrm{p}<0.0001$ for difference $)$. Linear regres- sion of digoxin level against date yielded an annual decline in mean digoxin level of $0.02 \mathrm{ng} / \mathrm{dl} /$ year in men and $0.01 \mathrm{ng} / \mathrm{dl} /$ year in women $(\mathrm{p}<0.0001$ for men and $\mathrm{p}=0.01$ for women, non-significant difference in rate between men and women).

\section{Outcomes}

Death rates after HF diagnosis without adjustment showed similar rates of mortality in all four cohorts defined by sex and digoxin use (table 2). 
Figure 1 Trends in drug use among (A) men and (B) women.
A Use of Common CHF Medications by Year in Men
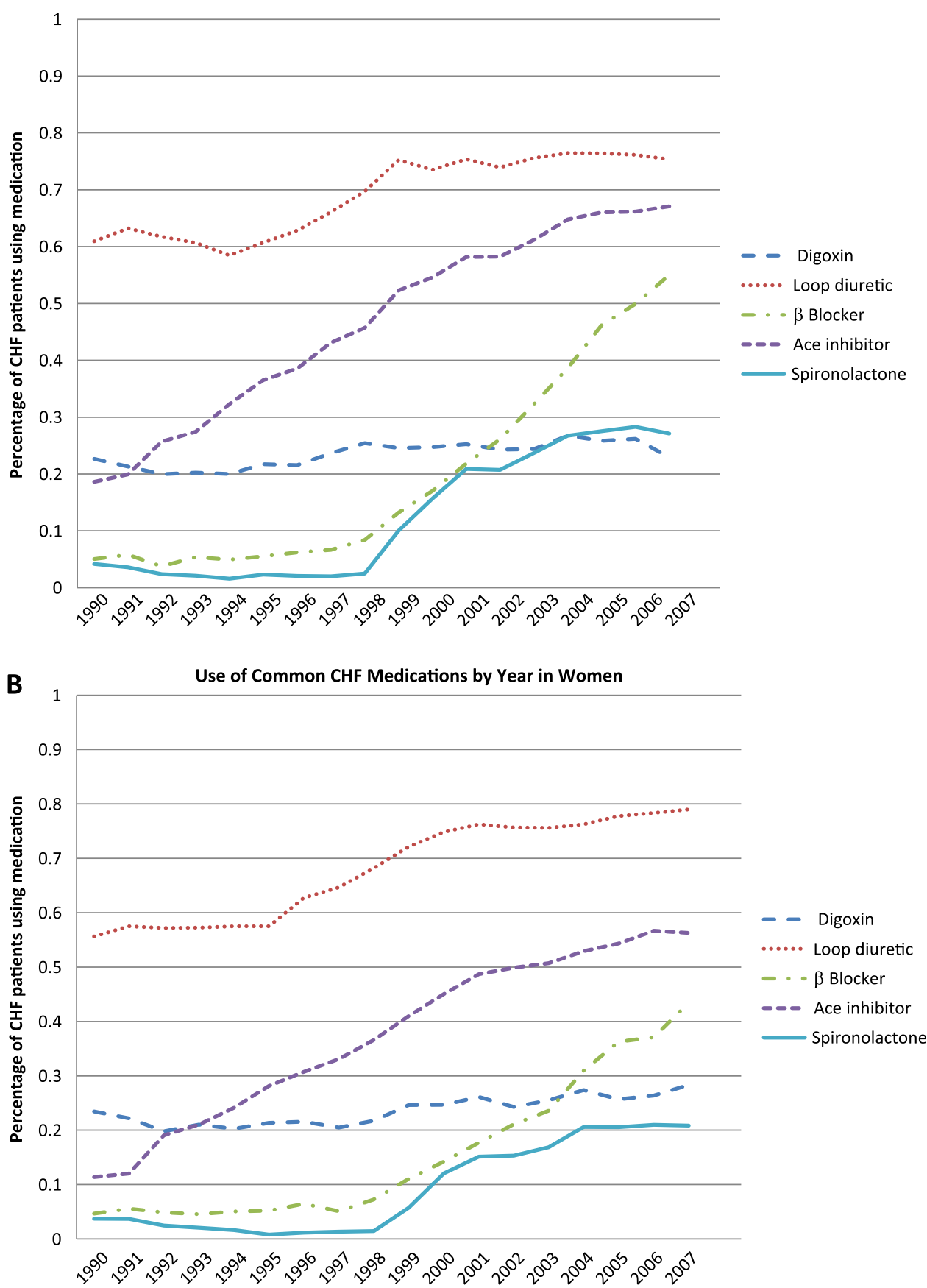

Multivariable modelling was pursued in stages (table 3). Briefly, we found that when universally available variables were adjusted for as covariates, there was no evidence of interaction between sex and digoxin use. The adjusted HR for digoxin exposure in a cohort restricted to women was 1.00 (95\% CI 0.96 to 1.06 ) and for a cohort restricted to men it was identical: 1.00 (95\% CI 0.95 to 1.06 ). A fully adjusted Cox proportional hazards model with a term for the interaction between digoxin use and sex yielded an interaction HR of 1.02

\begin{tabular}{lllll} 
Table 2 Event rates & & & $\begin{array}{c}\text { Deaths per } \\
\text { person-year }\end{array}$ \\
\hline Male digoxin non-user & N & Total person-years & Deaths & 4616 \\
Male digoxin user & 17707 & 53244 & 5076 & 0.09 \\
Female digoxin non-user & 19227 & 60648 & 2324 & 0.08 \\
Female digoxin user & 9487 & 24804 & 2782 & 0.09 \\
\hline
\end{tabular}


Table 3 Model with all universally available baseline variables

\begin{tabular}{|c|c|c|c|c|}
\hline & HR & Lower $\mathbf{C l}$ & Upper Cl & p Value \\
\hline Sex $\times$ digoxin interaction & 1.02 & 0.95 & 1.09 & 0.65 \\
\hline Digoxin use & 1.00 & 0.95 & 1.05 & 0.89 \\
\hline Female sex & 0.86 & 0.83 & 0.90 & $<0.0001$ \\
\hline Year of CHF diagnosis & 0.99 & 0.98 & 0.99 & $<0.0001$ \\
\hline Age $50-64$ years & 0.42 & 0.40 & 0.44 & $<0.0001$ \\
\hline Age $65-74$ years & 0.58 & 0.56 & 0.60 & $<0.0001$ \\
\hline Hypertension & 0.91 & 0.88 & 0.94 & $<0.0001$ \\
\hline Stroke & 1.34 & 1.29 & 1.40 & $<0.0001$ \\
\hline MI & 1.15 & 1.11 & 1.20 & $<0.0001$ \\
\hline COPD & 1.36 & 1.30 & 1.43 & $<0.0001$ \\
\hline Fracture & 1.08 & 1.03 & 1.14 & 0.001 \\
\hline Diabetes & 1.34 & 1.28 & 1.40 & $<0.0001$ \\
\hline Atrial fibrillation & 0.93 & 0.89 & 0.97 & 0.001 \\
\hline Loop diuretic use & 1.27 & 1.23 & 1.32 & $<0.0001$ \\
\hline $\begin{array}{l}\text { Potassium-sparing diuretic } \\
\text { use }\end{array}$ & 1.07 & 1.03 & 1.11 & 0.00 \\
\hline Thiazide diuretic & 1.03 & 0.99 & 1.07 & 0.16 \\
\hline Oral anticoagulant use & 0.92 & 0.87 & 0.98 & 0.001 \\
\hline$\beta$ Blocker use & 0.83 & 0.79 & 0.86 & $<0.0001$ \\
\hline ACE use & 0.97 & 0.93 & 1.00 & 0.07 \\
\hline ARB use & 0.90 & 0.82 & 0.98 & 0.01 \\
\hline CCB use & 0.93 & 0.90 & 0.97 & 0.001 \\
\hline
\end{tabular}

ARB, angiotensin receptor blocker; CCB, calcium channel blocker; COPD, chronic obstructive pulmonary disease; MI, myocardial infarction.

(95\% CI 0.95 to 1.09 ), where a HR significantly $>1$ would have indicated that digoxin use in women conferred a greater hazard of death than in men.

We examined whether the dose of digoxin mediated any interaction between sex and exposure by including digoxin dose in the model both as an independent variable and as an interaction term with sex, both as a categorical and a continuous variable. The same was done with serum digoxin levels. None of these analyses yielded any significant interaction terms. Low levels of digoxin in the serum $(<0.9 \mathrm{ng} / \mathrm{dl})$ were associated with lower mortality than higher levels (HR 0.71 , 95\% CI 0.67 to $0.76, p=0.007$ ), and this association did not differ by sex.

Sensitivity analyses including restriction of the cohort to persons actively taking loop diuretics, permitting use of data without the adjusted mortality ratio being up to standard, analysing the subset of the population with blood pressure, BMI and serum creatinine available, defining comorbid conditions based only on data from the 1 year preceding cohort entry, excluding patients with a diagnosis of atrial fibrillation and excluding persons at the time of cross-over to a different exposure category, all confirmed the results of the primary analysis, never showing a substantial or statistically significant interaction between sex and digoxin and mortality.

\section{DISCUSSION}

The primary finding from this study was the absence of a large interaction between digoxin use and sex affecting mortality, with a $95 \%$ CI excluding a HR $>1.09$ for the interaction. This suggests that the association between digoxin and mortality is similar in women and men. Sensitivity analyses did not affect this estimate materially.

The other prespecified aims of the study were to assess whether any interaction might be mediated by digoxin dose or by higher serum digoxin levels, both of which are thought to potentially affect mortality rates. ${ }^{3-7}$ Analyses incorporating digoxin dose and serum digoxin levels showed no evidence of such mediation. We did note an association between lower serum digoxin levels and lower mortality, but since such an association could easily be strongly confounded (eg, if more symptomatic patients were pushed to higher serum digoxin levels), this paper does not directly support or refute the claim that lower serum levels of digoxin lead to lower mortality risk.

An interesting incidental finding was rapid increases in the rates of $\beta$ blocker, ACE and spironolactone use beginning at the same time that major studies were published establishing that these drugs prevented mortality in HF. $^{16-18}$ Comparison by sex shows that while overall trends are similar, women consistently have lower levels of use of these life-saving agents compared with men.

A major strength of this study is its large sample size, permitting precise point estimates of associations. The size of the database also permitted numerous sensitivity analyses on subpopulations. The lack of interaction between sex and digoxin use was robust to adjustment for numerous potential confounders and to all sensitivity analyses. The major limitation of this study is that it is non-randomised. Although there is no evidence of substantial confounding of the main study result, confounding could still bias these results. Access to additional information that was not recorded in THIN, 
particularly the type (systolic vs diastolic) and severity of $\mathrm{HF}$, would have been helpful but only a randomised trial (ideally a series of such trials in various populations and dosing regimens) could definitively avoid concerns about confounding.

The outcome of this study is discordant with the post hoc analysis of the DIG trial ${ }^{2}$ but consistent with two prior observational studies that did not observe any digoxin-sex interaction. ${ }^{10}{ }^{11}$ It complements those prior studies by having over eight times their sample sizes and a more diverse cohort. Because the finding of an interaction from the DIG trial was the result of post hoc analysis, it is conceivable that the finding was a type 1 error (false positive), but it is also possible that the DIG trial results were correct, and the observational results are biased by unmeasured confounders that affected the interaction analysis. An interesting incidental finding of this study is that interventions known to reduce mortality in $\mathrm{HF}$ are used less in women than in men who carry that diagnosis (figure 1A,B). ${ }^{16-18}$ It would be premature to conclude that these differences necessarily imply worse care since there are important differences between the male and female populations with HF that might legitimately affect prescribing practices. However, the systematic differences in drug usage between the sexes deserve further investigation.

In conclusion, this study did not identify a difference between the sexes in the hazard of death associated with the use of digoxin. These results are of use in the context of a clinical question-whether digoxin can be used as safely in women as it can in men-for which there are few randomised data. These results suggest that this drug, with its proven ability to reduce the need for hospitalisation, is still a viable therapeutic option in women with HF.

\section{Author affiliations}

${ }^{1}$ Division of Endocrinology, Diabetes, and Metabolism in the Department of Medicine at New York-Presbyterian Hospital/Weill Cornell Medical Center, New York, New York, USA

${ }^{2}$ Center for Clinical Epidemiology and Biostatistics, Department of Biostatistics and Epidemiology, Perelman School of Medicine at the University of Pennsylvania, Philadelphia, Pennsylvania, USA

${ }^{3}$ Division of Pharmacoepidemiology and Pharmacoeconomics and Renal Division, Brigham and Women's Hospital, Harvard Medical School, Boston, Massachusetts, USA

${ }^{4}$ Section of Pulmonary and Critical Care, Dartmouth-Hitchcock Medical Center, Lebanon, New Hampshire, USA

${ }^{5}$ Outcome Sciences, A Quintiles Company, Cambridge, MA, USA

Contributors JHF is the guarantor of this manuscript, having been primarily responsible for study design, data analysis including programming and biostatistics and manuscript composition. JHF, BK, KH, SB, JM, CR, BLS and $\mathrm{SH}$ all made substantial contributions to the conception and design of this study. JHF was primarily responsible for analysis and interpretation of the data, but all listed authors made significant contributions to this as well. All the listed authors made substantial contributions in drafting the article or revising it critically for important intellectual content.
Funding FOCUS Medical Student Fellowship in Women's Health supported by the Edna G. Kynett Memorial Foundation, Pharmacoepidemiology Training Funds donated to the Center for Clinical Epidemiology and Biostatistics of the University of Pennsylvania School of Medicine. The study sponsors had no influence over design of the study, the handling of data, the composition of the manuscript or the decision to submit it.

Competing interests SH has consulted for Abbott Laboratories. BLS has consulted for Abbott Laboratories, received payment for lectures from Teva Pharmaceutical Industries and has consulted for Teva and for GlaxoSmithKline. All authors on this manuscript have a connection to a training programme that has received funding from Abbott Laboratories and GlaxoSmithKline. None of these connections related directly to digoxin. These companies are among the over 50 corporations that manufacture or distribute digoxin.

Provenance and peer review Not commissioned; externally peer reviewed.

Data sharing statement No additional data available.

\section{REFERENCES}

1. The Digitalis Investigation Group. The effect of digoxin on mortality and morbidity in patients with heart failure. $N$ Engl J Med 1997;336:525-33.

2. Rathore SS, Wang $\mathrm{Y}$, Krumholz HM. Sex-based differences in the effect of digoxin for the treatment of heart failure. $N$ Engl $J$ Med 2002;347:1403-11.

3. Adams KF Jr, Patterson JH, Gattis WA, et al. Relationship of serum digoxin concentration to mortality and morbidity in women in the digitalis investigation group trial: a retrospective analysis. J Am Coll Cardiol 2005;46:497-504.

4. Rathore SS, Curtis JP, Wang Y, et al. Association of serum digoxin concentration and outcomes in patients with heart failure. JAMA 2003;289:871-8.

5. Ahmed A, Rich MW, Love TE, et al. Digoxin and reduction in mortality and hospitalization in heart failure: a comprehensive post hoc analysis of the DIG trial. Eur Heart J 2006;27:178-86.

6. Gheorghiade M, van Veldhuisen DJ, Colucci WS. Contemporary use of digoxin in the management of cardiovascular disorders. Circulation 2006;113:2556-64.

7. Brophy JM. Rehabilitating digoxin. Eur Heart $J$ 2006;27:127-9.

8. Haynes K, Heitjan D, Kanetsky P, et al. Declining public health burden of digoxin toxicity from 1991 to 2004. Clin Pharmacol Ther 2008;84:90-4.

9. Morris SA, Hatcher HF, Reddy DK. Digoxin therapy for heart failure: an update. Am Fam Physician 2006;74:613-18.

10. Domanski M, Fleg J, Bristow $\mathrm{M}$, et al. The effect of gender on outcome in digitalis-treated heart failure patients. $J$ Card Fail 2005;11:83-6.

11. Andrey JL, Romero S, García-Egido A, et al. Mortality and morbidity of heart failure treated with digoxin. A propensity-matched study. Int $J$ Clin Pract 2011;65:1250-8.

12. Lewis JD, Schinnar R, Bilker WB, et al. Validation studies of the health improvement network (THIN) database for pharmacoepidemiology research. Pharmacoepidemiol Drug Saf 2007; 16:393-401.

13. Maguire A, Blak BT, Thompson M. The importance of defining periods of complete mortality reporting for research using automated data from primary care. Pharmacoepidemiol Drug Saf 2009;18:76-83.

14. Austin PC. Balance diagnostics for comparing the distribution of baseline covariates between treatment groups in propensity-score matched samples. Stat Med 2009;28:3083-107.

15. Cox DR. Regression models and life-tables (with discussion). J Roy Statist Soc Ser B 1972;34:187-220.

16. Anon. Effect of metoprolol $\mathrm{CR} / \mathrm{XL}$ in chronic heart failure: Metoprolo CR/XL Randomised Intervention Trial in Congestive Heart Failure (MERIT-HF). Lancet 1999;353:2001-7.

17. The SOLVD Investigators. Effect of enalapril on survival in patients with reduced left ventricular ejection fractions and congestive heart failure. N Engl J Med 1991;325:293-302.

18. Pitt B, Zannad F, Remme WJ, et al. The effect of spironolactone on morbidity and mortality in patients with severe heart failure. Randomized Aldactone Evaluation Study Investigators. N Engl J Med 1999;341:709-17. 


\section{Correction}

Flory JH, Ky B, Haynes K, et al. Observational cohort study of the safety of digoxin use in women with heart failure. BMJ Open 2012;2: e000888. The fourth author in this article should be listed as Brunelli SM (not S Brunelli M).

BMJ Open 2012;00:e000888corr1. doi:10.1136/bmjopen-2012-000888corr1 